\title{
Using Internationalisation at Home to provide international learning to all physiotherapy students
}

T Dahl-Michelsen, ${ }^{1}$ BSc Physiotherapy, MSc Health Sciences, PhD; K S Groven, ${ }^{1}$ BSc Physiotherapy, MSc Health Sciences, PhD; M Rowe, ${ }^{2}$ BSc Physiotherapy, MSc Physiotherapy, $\mathrm{PhD}$

${ }^{1}$ Department of Physiotherapy, Faculty of Health Sciences, OsloMet-Oslo Metropolitan University, Norway

${ }^{2}$ Department of Physiotherapy, Faculty of Community and Health Sciences, University of the Western Cape, Cape Town, South Africa

Corresponding author: T Dahl-Michelsen (tonedami@oslomet.no)

\section{Why was the idea necessary?}

The recent emphasis on globalisation in healthcare education includes the importance of internationalisation as an approach for providing students with international and intercultural competence. ${ }^{[1]}$ However, the challenge of sending large groups of students on exchange programmes has driven the rise of Internationalisation at Home $(\mathrm{IaH})$ programmes, where all students are given international learning opportunities. ${ }^{[2]}$ This article elaborates on how IaH provided international learning opportunities to 104 physiotherapy students in Norway and South Africa (SA). The article also highlights how the project influenced students' understanding of the local context in clinical practice and rehabilitation.

\section{What was tried?}

In 2017, we piloted an IaH project that included all physiotherapy students in the second-year cohort at Oslo Metropolitan University in Norway and the University of the Western Cape in Cape Town, SA. The project included online and face-to-face components, where students completed an assignment and then received feedback and guidance from peers in the other institution. The assignment was informed by principles of photovoice methodology, ${ }^{[3]}$ where students took photos of their local communities with the aim of contextualising healthcare and patient experiences of health and rehabilitation services. These photos were discussed in class and then combined with personal reflections that emphasised how students' thinking regarding clinical practice and rehabilitation was influenced by their local context. Students were also expected to integrate relevant academic literature with their personal narratives to better understand their reflections. The assignment was mandatory for all students as part of their course. We found that most students were eager to participate and enjoyed the assignment, with high levels of activity throughout.

The initial drafts of the assignment were shared on Google Drive so that students from both universities could provide one another with guidance and critical input in the form of questions. This feedback was then reviewed by lecturers, who provided additional comments where necessary. Students were able to make changes to their assignments using the peer feedback before submitting the final version. All students participated in a face-toface seminar, where lecturers in each university guided a discussion on how local health contexts influenced clinical practice and health systems more generally. Finally, we conducted a focus group discussion with 4 students from the Norwegian cohort, during which they elaborated further on the themes from the seminar. The SA students chose not to participate in the focus group interview because the interviews took place during the students examination period.

\section{What were the lessons learnt?}

When students gave each other feedback, they not only learnt about other health contexts but also gained insight into their taken-for-granted assumptions regarding their own health system. They were able to identify some of the social and cultural influences on healthcare and rehabilitation, as well as specific aspects of their own culture that were previously underappreciated.

This pilot study demonstrates a process for implementing low-cost $\mathrm{IaH}$ projects, where students in very different health, social and cultural contexts can be introduced to internationalisation concepts within the existing curriculum. Such programmes may have important implications for countries that are interested in developing internationalisation components in the curriculum, but cannot afford to send large groups of students on exchange programmes. The availability of free online environments that facilitate student interaction, in combination with common learning resources, means that internationalisation can be achieved without expensive and environmentally harmful travel.

\section{Declaration. None.}

Acknowledgements. The authors extend their appreciation and gratitude to the student participants.

Author contributions. TD-M and MR collaboratively designed the project. All three authors participated in writing up the article.

Funding. None.

Conflicts of interest. None.

Deardorff DK, Arasaratnam-Smith LA, eds. Intercultural Competence in Higher Education: Internation Approaches, Assessment and Application. Abingdon, UK: Routledge, 2017.

Ryan J, ed. Cross-cultural Teaching and Learning for Home and International Students: Internationalisation of Pedagogy and Curriculum in Higher Education. Abingdon, UK: Routledge, 2012.

3. Sutton-Brown CA. Photovoice: A methodological guide. Photography Culture 2014;7(2):169-185. https://doi.org/10 2752/175145214X13999922103165

Accepted 6 November 2019.

Afr J Health Professions Educ 2020;12(1):5. https://doi.org/10.7196/AJHPE.2020.v12i1.1260 